\title{
TITLE:
}

\section{Induced top-Yukawa coupling and suppressed Higgs mass parameters}

$\operatorname{AUTHOR}(\mathrm{S})$ :

Kobayashi, T; Nakano, H; Terao, H

\section{CITATION:}

Kobayashi, T ...[et al]. Induced top-Yukawa coupling and suppressed Higgs mass parameters. PHYSICAL REVIEW D 2005, 71(11): 115009.

ISSUE DATE:

2005-06

URL:

http://hdl.handle.net/2433/50538

RIGHT:

Copyright 2005 American Physical Society 
PHYSICAL REVIEW D 71, 115009 (2005)

\title{
Induced top-Yukawa coupling and suppressed Higgs mass parameters
}

\author{
Tatsuo Kobayashi* \\ Department of Physics, Kyoto University, Kyoto 606-8502, Japan \\ Hiroaki Nakano ${ }^{\dagger}$ \\ Department of Physics, Niigata University, Niigata 950-2181, Japan \\ Haruhiko Terao \\ Institute for Theoretical Physics, Kanazawa University, Kanazawa 920-1192, Japan
}

(Received 1 February 2005; published 21 June 2005)

\begin{abstract}
In the scenarios with heavy top squarks, mass parameters of the Higgs field must be fine-tuned due to a large logarithmic correction to the soft scalar mass. We consider a new possibility that the top-Yukawa coupling is small above $\mathrm{TeV}$ scale. The large top mass is induced from strong Yukawa interaction of the Higgs with another gauge sector, in which supersymmetry breaking parameters are given to be small. Then it is found that the logarithmic correction to the Higgs soft scalar mass is suppressed in spite of the strong coupling, and the fine-tuning is ameliorated. We propose an explicit model coupled to a superconformal gauge theory which realizes the above situation.
\end{abstract}

DOI: 10.1103/PhysRevD.71.115009

PACS numbers: 12.60.Jv, 14.65.Ha, 14.80.Bn

\section{INTRODUCTION}

Supersymmetric little hierarchy $[1,2]$ means a large discrepancy between the scale of weak boson masses and the scale of supersymmetry breaking masses, specially for the Higgs fields. In the minimal supersymmetric standard model (MSSM), the minimization condition for the potential of neutral Higgs components is given by

$$
\frac{M_{z}^{2}}{2}=-\mu^{2}-m_{H_{u}}^{2}
$$

for a moderate value of $\tan \beta$. Here $m_{H_{u}}$ denotes the soft supersymmetry breaking mass for the up-type Higgs field $H_{u}$, while $\mu$ denotes the supersymmetric mass parameter. If $\left|m_{H_{u}}\right|$ is much larger than $M_{z}$, the two mass parameters $m_{H_{u}}$ and $\mu$ must be fine-tuned so as to nearly cancel out each other. However there is no theoretical ground that low energy values of these parameters are related mutually. Therefore, it seems to be natural for the Higgs mass parameters, $\left|m_{H_{u}}\right|$ and $|\mu|$, to appear less than a few hundred $\mathrm{GeV}$.

Contrary to this, the soft supersymmetry breaking mass $\left|m_{H_{u}}\right|$ appears to be fairly large at low energy in the most supersymmetric models. So this is sometimes called the supersymmetric little hierarchy problem. In this article we consider a way out of this problem by some extension of the MSSM. The problem originates in the large radiative correction to the soft scalar mass of $H_{u}$, which is given explicitly at one-loop level by

\footnotetext{
*Electronic address: kobayash@gauge.scphys.kyoto-u.ac.jp

${ }^{\dagger}$ Electronic address: nakano@muse.sc.niigata-u.ac.jp

${ }^{\ddagger}$ Electronic address: terao@hep.s.kanazawa-u.ac.jp
}

$$
\delta m_{H_{u}}^{2} \sim-\frac{12}{16 \pi^{2}} Y_{t}^{2} m_{\tilde{t}}^{2} \ln \frac{\Lambda}{m_{\tilde{t}}}
$$

Here $Y_{t}$ and $m_{\tilde{t}}$ denote the top-Yukawa coupling and the soft supersymmetry breaking mass of stop (top sparticle). We represent an uppermost scale of the MSSM by $\Lambda$, which may be taken to be the grand unified theory (GUT) scale; $\Lambda \simeq 10^{16} \mathrm{GeV}$. Then the correction $\left|\delta m_{H_{u}}\right|$ is found to be comparatively larger than $m_{\tilde{t}}$ because of the sizable top-Yukawa coupling $Y_{t}$. This negative correction to the Higgs scalar mass squared gives rise to radiative electro-weak symmetry breaking (EWSB) [3], which is thought to be one of the beautiful features of the MSSM. However the problem is that the radiative correction is too large, since $m_{\tilde{t}}$ is supposed to be more than several hundred $\mathrm{GeV}$ for the following reasons.

First, one is based on the experimental lower bound of the lightest Higgs boson mass $m_{h^{0}}$, which is $114 \mathrm{GeV}$ [4]. On the other hand, the MSSM predicts the lightest $C P$-even Higgs boson mass $m_{h^{0}}$ to be less than $M_{Z}$ at the tree level. This discrepancy can be covered by sizable radiative correction for $m_{h^{0}}$, when the top-stop mass splitting is relatively large [5]. Explicitly, the one-loop correction may be written roughly as

$$
\Delta m_{h^{0}}^{2} \sim \frac{3}{4 \pi^{2}} Y_{t}^{2} m_{t}^{2} \ln \left(\frac{m_{\tilde{t}}^{2}}{m_{t}^{2}}\right),
$$

where $m_{t}$ denotes the top mass. This formula requires $m_{\tilde{t}} \geq$ $500 \mathrm{GeV}$ so as for the lightest Higgs mass in the MSSM to satisfy the experimental bound. Then the soft supersymmetry breaking mass $\left|m_{H_{u}}\right|$ is supposed to be more than about $800 \mathrm{GeV}$ in the MSSM, and rather tight fine-tuning less than a few percent is required in order to satisfy Eq. (1). 
Second, one is theoretical. One of strong supports for the low energy supersymmetry is gauge coupling unification around the scale $\Lambda \sim 2 \times 10^{16} \mathrm{GeV}$. When the gaugino masses $M_{a}(a=1,2,3)$ are also unified around $\Lambda$, the gaugino masses enjoy the famous GUT relation with the corresponding gauge coupling $g_{a}$ as $M_{a} / g_{a}^{2}=$ const. Therefore, the gauge coupling unification leads to a rather heavy gluino mass at low energy. This is the same for the minimal supergravity (mSUGRA) model [2]. Such a gluino mass enhances the soft supersymmetry breaking masses of squarks at low energy through radiative corrections. Explicitly the correction to $m_{\tilde{t}}^{2}$ becomes as large as $(500 \mathrm{GeV})^{2}$, even if $M_{1} \sim 100 \mathrm{GeV}$ at low energy. Then the soft mass of Higgs receives large radiative corrections through these heavy squarks. Thus we may say that the primary origin of the fine-tuning problem is the enhanced gluino mass.

The same fine-tuning problem arises in the models with the so-called gauge mediated supersymmetry breaking (GMSB), which is one of the most popular scenarios explaining the flavor universal soft masses. There the soft masses and also the gaugino masses are generated at some lower scale than the GUT scale. However the size of the generated squarks masses as well as the gaugino masses are found to be large as those given in the mSUGRA scenario. Thus the fine-tuning problem is found to be rather severe also in the GMSB [6].

In this article, therefore, we consider scenarios, in which squarks masses are large but the top-Yukawa coupling $Y_{t}$ is small above $\mathrm{TeV}$ scale. Then the radiative correction given by Eq. (3) is reduced obviously. In order to explain our motivation to study such scenarios, let us first mention the recent proposals to remedy this fine-tuning problem by various extensions of the MSSM. Many of them are to enhance the effective quartic coupling of the Higgs field in the low energy theory. The ways are various; low energy supersymmetry breaking [7], additional F-term contributions [8], additional D-term contributions [9], an additional sizable Yukawa coupling of the Higgs field with extra fields [10]. Indeed enhancement of the quartic coupling may improve degree of the fine-tuning somewhat, however this effect is not so significant. Rather it is essential that the tree-level value of the lightest Higgs boson mass is enhanced, and the large stop mass is not required any more to satisfy the experimental bound. Then the radiative correction (2) to the Higgs soft mass may be sufficiently reduced in the case of light stop masses. Thus it should be noted that light gluino mass as well as the light stop mass must be assumed in these scenarios, which does not conform to the sparticle spectra obtained in the frameworks of mSUGRA nor GMSB.

Therefore it would be more interesting to study the models in which only the Higgs mass parameters are suppressed with keeping the other soft masses heavy, as given in the GMSB scenario. In order to realize such a situation, it is necessary to eliminate or suppress the logarithmic correction to the Higgs soft mass, $\delta m_{H_{u}}^{2}$, which is given by Eq. (2) approximately. Actually there have been proposed only a few scenarios along this line of thought. One way is given by the so-called supersoft supersymmetry breaking mechanism [11]. There the soft masses are generated only through finite loop diagrams so that the flavor universality of them is automatically guaranteed. On top of that, the soft scalar mass of Higgs is free from logarithmic enhancement and is also suppressed by one-loop factor. Another one is the supersymmetric little Higgs model [12], in which the Higgs fields are assumed to be pseudo Nambu-Goldstone bosons, and their mass parameters are generated only radiatively. Then the enlarged symmetry ensures that the logarithmic corrections to the mass parameter appear at the two-loop level and, therefore, are suppressed.

Two of the authors also presented models in which the Higgs field has an additional Yukawa coupling with a superconformal gauge sector [13]. It has been known $[14,15]$ that soft masses introduced to the chiral matter fields of a superconformal gauge theory enjoy sum rules at infrared (IR) irrespectively of their values at high energy scale. In the previous paper, it was shown that the Higgs soft mass can be made finite and ultraviolet insensitive by using the sum rules. Moreover, the obtained Higgs mass is one-loop suppressed. Besides the Higgs field acquires a large anomalous dimension and, therefore, the $\mu$ parameter is suppressed at the same time. Thus both of the mass parameters of the Higgs field turn out to be small at low energy by the same dynamics and become free from finetuning. However the explicit model relies upon assumptions to unknown dynamics in order to realize the finite soft masses and may be somewhat artificial. Besides that, it is an undesirable feature that top-Yukawa coupling is suppressed at low energy due to the large anomalous dimension of the Higgs field. ${ }^{1}$

In this paper we seek for another possibility that the Higgs field in the MSSM is sequestered from gluino and top squarks, whose large masses cause the fine-tuning problem. We suppose that the top-Yukawa coupling is small above $\mathrm{TeV}$ scale. Then the radiative correction given in Eq. (2) is suppressed and becomes harmless. This radical assumption also requires a new source for the top mass in place of the top-Yukawa coupling. So we introduce a strongly coupled gauge sector and a new Yukawa interaction between the Higgs field and the matter fields in the strongly coupled sector. The large top mass is found to be induced at low energy through mixing between the fundamental top quarks and the strongly coupled matter fields.

\footnotetext{
${ }^{1}$ Recently Luty and Okui [16] also considered suppression of the Higgs mass parameters by use of conformal dynamics in nonsupersymmetric case in order to solve the hierarchy problem. There also the top-Yukawa coupling appears to be suppressed at low energy.
} 
One might wonder that the new Yukawa coupling induces a large correction to the Higgs mass parameter just as the top-Yukawa coupling does, and is not helping to solve the problem. However if the supersymmetry breaking parameters in the strong gauge sector are sufficiently small for some reason, then the Higgs mass is not enhanced through the new Yukawa coupling. Here it is noted that if the strong gauge theory is superconformal, then the gaugino mass and the A-parameters necessarily vanish. Later we will give an explicit model in which the Higgs mass is preserved to be small due to the superconformal dynamics. We stress that the superconformal dynamics itself does not suppress the soft scalar mass of Higgs, and the scenario considered here is distinct from the previous one considered in Ref. [13].

The article is organized as follows. In section II we explain the basic mechanism for sequestering Higgs from the large soft masses of gluino and stops by assuming small top-Yukawa coupling above $\mathrm{TeV}$ scale. We present an explicit model using superconformal dynamics in section III. There, the mixing between top quarks and the extra matter fields, which induces large top mass, is also shown. In section IV we study some phenomenological aspects of the model given in section III. Finally section V is devoted to conclusion and discussion.

\section{SEQUESTERING HIGGS FROM LARGE SUPERSYMMETRY BREAKING}

First let us discuss renormalization properties of the soft masses of Higgs $H_{u}$ and stop fields $Q_{3}, \bar{u}_{3}$ in the MSSM at one-loop level. We neglect the bottom Yukawa coupling $Y_{b}$ and the gauge interactions other than $S U(3)_{C}$, as these effects are not significant for the fine-tuning problem. Then the renormalization group (RG) equations for the soft masses are given by

$$
\begin{gathered}
16 \pi^{2} \frac{d m_{Q_{3}}^{2}}{d \ln \mu}=X_{t}-\frac{32}{3} g_{3}^{2}\left|M_{3}\right|^{2}, \\
16 \pi^{2} \frac{d m_{\bar{u}_{3}}^{2}}{d \ln \mu}=2 X_{t}-\frac{32}{3} g_{3}^{2}\left|M_{3}\right|^{2}, \quad 16 \pi^{2} \frac{d m_{H_{u}}^{2}}{d \ln \mu}=3 X_{t},
\end{gathered}
$$

where $X_{t}$ denotes the following combination of the soft scalar masses and the A-parameter $A_{t}$;

$$
X_{t}=2\left|Y_{t}\right|^{2}\left[\left(m_{Q_{3}}^{2}+m_{\bar{u}_{3}}^{2}+m_{H_{u}}^{2}\right)+\left|A_{t}\right|^{2}\right] \text {. }
$$

The parameter $A_{t}$ follows the RG equation given by

$$
16 \pi^{2} \frac{d A_{t}}{d \ln \mu}=2\left|Y_{t}\right|^{2} A_{t}+\frac{32}{3} g_{3}^{2} M_{3}
$$

The large gluino mass $M_{3}$ induces large corrections not only to squark masses $m_{Q_{3}}^{2}$ and $m_{\bar{u}_{3}}^{2}$ but also to $A_{t}$. However if the top-Yukawa coupling $Y_{t}$ is given to be small enough, then the soft mass parameter of up-type Higgs $m_{H_{u}}^{2}$ is protected from the corrections due to the gluino and stop masses. That is the scenario we consider in this paper.

We note also that these RG equations show an interesting property as follows. If we drop off $M_{3}$ and $A_{t}$ from these equations and treat $Y_{t}$ as a constant approximately, then these coupled equations are easily solved. There are two constant modes for $\left(m_{Q_{3}}^{2}, m_{\bar{u}_{3}}^{2}, m_{H_{u}}^{2}\right)$, which are proportional to $(1,-1,0)$ and $(1,1,-2)$. The linearly independent mode proportional to $(1,2,3)$ is suppressed towards IR with a power of scale. ${ }^{2}$ Therefore the soft masses satisfy the sum rule; $m_{Q_{3}}^{2}+m_{\bar{u}_{3}}^{2}+m_{H_{u}}^{2} \rightarrow 0$ at low energy. Here it is noted that the soft masses are not enhanced from the high energy values. As long as initial values of the scalar masses are given to be small, $\left|m_{H_{u}}\right|$ is always small at low energy. Thus the supersymmetric little hierarchy may be ameliorated by suppressing the gluino mass and the A-parameter for the top-Yukawa coupling compared with other gaugino masses. In this paper we do not pursuit for this possibility keeping scenarios of mSUGRA and GMSB in our mind.

Now, let us study the scenario with small top-Yukawa coupling $Y_{t}$ e.g. as $Y_{b}$, in order to sequester the Higgs field from large radiative corrections due to the gluino and stop masses, as said above. However, in this scenario, we need to explain the large top mass by another mechanism. As was mentioned in section I, we introduce an extra strongly interacting gauge theory with the gauge group $G_{S}$. We also assume additional chiral superfields $(\Phi, \bar{\phi})$, which are charged under $G_{S}$. The quark fields $\left(Q_{3}, \bar{u}_{3}\right)$ are charged under $S U(3)_{C}^{\prime}$, while $(\Phi, \bar{\phi})$ are singlet. We consider their Yukawa interaction with $H_{u}$ as

$$
W \sim Y_{t} Q_{3} \bar{u}_{3} H_{u}+\lambda \Phi \bar{\phi} H_{u}
$$

The new Yukawa coupling $\lambda$ becomes large at low energy due to strong gauge interaction. It is noted that large $\lambda$ reduces $Y_{t}$. In practice we consider scenarios in which the symmetry $G_{S} \times S U(3)_{C}^{\prime}$ is spontaneously broken to the color gauge group $S U(3)_{C}$ at low energy. After this symmetry breaking, $\Phi$ and $\bar{\phi}$ carry the same charges as $Q_{3}$ and $\bar{u}_{3}$, respectively. Therefore these extra matter fields can mix with the original top quarks. Eventually large topYukawa coupling is induced from the second term of the superpotential given in (7) in the low energy effective

\footnotetext{
${ }^{2}$ We can always tune the high energy values of the scalar masses so that $\left|m_{H_{u}}\right|$ vanishes at low energy scale. Such initial values are given as two parameter solutions. Accidentally the universal initial values given at GUT scale happen to lead to vanishing $\left|m_{H_{u}}\right|$ at the EW scale, which is known as the focus point [17]. However natural explanation for the initial values is required in turn.
} 
theory. We will explain how the mixing can be generated by presenting an explicit model in the next section.

We also assume that the extra matter fields have their vectorlike partners $(\bar{\Phi}, \phi)$ and supersymmetric mass terms like $\mu$-term of the Higgs fields;

$$
W \sim \mu H_{u} H_{d}+M_{\Phi} \Phi \bar{\Phi}+M_{\phi} \phi \bar{\phi} .
$$

We will explicitly see later that $\mu$ is suppressed, while $M_{\Phi}$ and $M_{\phi}$ are enhanced by the strong extra gauge interaction [13]. Therefore we may suppose that the decoupling scale given $M_{\Phi}$ or $M_{\phi}$ to be about several TeV. We also assume that the symmetry breaking takes place around this scale.

How about the radiative correction to the soft mass of $H_{u}$ ? What we should point out are corrections induced by the large extra Yukawa coupling $\lambda$. Here it should be noted that the RG equations for soft scalar masses $m_{\Phi}^{2}, m_{\bar{\phi}}^{2}$ and $m_{H_{u}}^{2}$ have the same structure as in Eqs. (4). Therefore, if the gaugino mass of the extra gauge theory $M_{S}$ and also the Aparameter for the extra trilinear interaction $A_{\lambda}$ are both suppressed well, then the soft scalar mass of Higgs $m_{H_{u}}^{2}$ is not enhanced at all in spite of the strong couplings. In such a case, the mass parameters of Higgs are fairly smaller than the gluino mass at low energy, as long as $m_{\Phi}^{2}$ and $m_{\bar{\phi}}^{2}$ given at high energy scale are also small. Below the scale of symmetry breaking, $m_{H_{u}}^{2}$ receives the negative correction through the effective top-Yukawa coupling just as in the MSSM. However size of this correction is reduced by about one order, since $\Lambda$ in Eq. (2) is now given to be only several TeV. Thus radiative EWSB still occurs but the Higgs mass parameters appearing in Eq. (1) are suppressed well.

Now we discuss the ways to realize the above situation. Indeed there are at least two possibilities in which the above situation is realized naturally. The first one is an application of superconformal gauge theories. We note that both of the gaugino mass and the A-parameters are suppressed in proportion to certain powers of the renormalization scale in any superconformal gauge theories [14,15]. The superconformal gauge theories are not special at all. Only if the number of charged matter fields is given in the so-called conformal window, the gauge coupling is found to have an IR attractive fixed point [18], where the theory becomes conformal invariant. We will give somewhat detailed discussion of this in section III. Thus in the case that the extra gauge sector becomes superconformal with approaching an IR fixed point, the Higgs field can be sequestered from supersymmetry breaking effects. In the next section we will present an explicit model suppressing the Higgs mass parameters by the superconformal dynamics.

Alternatively we may consider the GMSB whose messengers are singlet under the extra gauge interaction. Then the gaugino of the strong sector is massless from the beginning and therefore the A-parameter is not enhanced. In addition, the soft scalar masses of the extra matter fields are as small as slepton masses. Explicit considerations of such GMSB scenarios will be reported elsewhere.

\section{HIGGS COUPLED WITH A SUPERCONFORMAL SECTOR}

In this section we consider an explicit model in which the Higgs field couples with a superconformal gauge theory through a new Yukawa coupling. First we assume the extra gauge group $G_{S}$ to be $S U(3)_{\mathrm{SC}}$, and the color $S U(3)_{C}$ of the standard model is given by the diagonal subgroup of $S U(3)_{\mathrm{SC}} \times S U(3)_{C}^{\prime}$. We introduce vectorlike matter fields charged under $S U(3)_{\mathrm{SC}}$ so that there is an IR fixed point with strong gauge coupling. Then the gauge couplings of these groups are related with $1 / g_{3}^{2}=1 / g_{\mathrm{SC}}^{2}+1 / g_{3}^{2}$. Therefore the gauge coupling of $S U(3)_{C}^{\prime}$ is almost the same as the color gauge coupling, $g_{3} \sim g_{3}^{\prime}$, since $g_{\mathrm{SC}}$ is as large as $4 \pi$ at the fixed point. The gaugino mass at low energy, $M_{3}$, is related with the gaugino masses $M_{\mathrm{SC}}$ and $M_{3}^{\prime}$ of $S U(3)_{\mathrm{SC}}$ and $S U(3)_{C}^{\prime}$ sectors by

$$
\frac{M_{3}}{g_{3}^{2}}=\frac{M_{\mathrm{SC}}}{g_{\mathrm{SC}}^{2}}+\frac{M_{3}^{\prime}}{g_{3}^{2^{\prime}}} .
$$

It is seen that $M_{3} \sim M_{3}^{\prime}$, since $M_{\mathrm{SC}}$ is suppressed by the superconformal dynamics.

The matter contents other than the fields of the MSSM are as follows;

\begin{tabular}{l|ccccc} 
& $S U(3)_{\mathrm{SC}}$ & $S U(3)_{C}^{\prime}$ & $S U(2)_{W}$ & $U(1)_{Y}$ & $R$ \\
\hline$\Phi$ & 3 & 1 & 2 & $1 / 6$ & - \\
$\bar{\Phi}$ & $3^{*}$ & 1 & 2 & $-1 / 6$ & - \\
$\phi$ & 3 & 1 & 1 & $2 / 3$ & - \\
$\bar{\phi}$ & $3^{*}$ & 1 & 1 & $-2 / 3$ & - \\
$\Omega$ & 3 & $3^{*}$ & 1 & 0 & + \\
$\bar{\Omega}$ & $3^{*}$ & 3 & 1 & 0 & +
\end{tabular}

Here $R$ denotes the R-parity. Quarks in the MSSM belong to the fundamental representation of $S U(3)_{C}^{\prime}$. The extra matter fields are not combined into representations of an $S U(5)$ because of their $U(1)_{Y}$ charge assignment. We will see later that gauge coupling unification is slightly modified.

The matter fields charged under $S U(3)_{\mathrm{SC}}$ are vectorlike and the number of the "flavor" $N_{f}$ is 6 . Therefore this gauge theory belongs to the conformal window given by $3 / 2 N_{c}<N_{f}<3 N_{c}$ and has an IR attractive fixed point. If we neglect the gauge couplings of $S U(3)_{C}^{\prime}, S U(2)_{W}$ and $U(1)_{Y}$, the anomalous dimensions of the vectorlike matter fields are exactly given by $\gamma_{\Phi}=\gamma_{\phi}=\gamma_{\Omega}=\left(3 N_{C}-\right.$ $\left.N_{f}\right) / N_{c}=-1 / 2$ at the fixed point.

These negative anomalous dimensions mean that the dimensions of the charged matter fields are less than the canonical ones $(d=1 / 2)$. So the Yukawa term $\lambda \Phi \bar{\phi} H_{u}$, which is allowed by the symmetry, is a dimension two operator at the fixed point of the pure gauge theory. Therefore this perturbation is relevant and the coupling $\lambda$ grows towards IR with a single power of the scale. However the large Yukawa coupling $\lambda$ increases the di- 
mension of the Higgs $H_{u}$, and is expected to eventually approach a new fixed point $\lambda_{*}$.

Though existence of this new fixed point has not been proven yet, we may demonstrate it by applying one-loop anomalous dimensions in the exact RG equations [19]. By neglecting the MSSM couplings, the anomalous dimensions are found to be

$$
\begin{aligned}
& \gamma_{\Phi}=-\frac{8}{3} \alpha_{\mathrm{SC}}+\alpha_{\lambda}, \quad \gamma_{\bar{\phi}}=-\frac{8}{3} \alpha_{\mathrm{SC}}+2 \alpha_{\lambda}, \\
& \gamma_{H_{u}}=3 \alpha_{\lambda}, \quad \gamma_{\bar{\Phi}}=\gamma_{\phi}=\gamma_{\Omega}=\gamma_{\bar{\Omega}}=-\frac{8}{3} \alpha_{\mathrm{SC}},
\end{aligned}
$$

where $\alpha_{\mathrm{SC}}=g_{\mathrm{SC}}^{2} / 8 \pi^{2}$ and $\alpha_{\lambda}=|\lambda|^{2} / 8 \pi^{2}$. The exact beta functions are given in terms of these anomalous dimensions as

$$
\begin{gathered}
\frac{d \alpha_{\mathrm{SC}}}{d \ln \mu}=-\frac{\alpha_{\mathrm{SC}}^{2}}{1-3 \alpha_{\mathrm{SC}}}\left(3+\gamma_{\Phi}+\frac{1}{2} \gamma_{\bar{\phi}} \gamma_{\bar{\Phi}}+\frac{1}{2} \gamma_{\phi}\right. \\
\left.+\frac{3}{2} \gamma_{\Omega}+\frac{3}{2} \gamma_{\bar{\Omega}}\right), \\
\frac{d \alpha_{\lambda}}{d \ln \mu}=\alpha_{\lambda}\left(\gamma_{\Phi}+\gamma_{\bar{\phi}}+\gamma_{H_{u}}\right) .
\end{gathered}
$$

Immediately the fixed point couplings are found to be

$$
\begin{gathered}
(\mathrm{A}):\left(\alpha_{\mathrm{SC}}^{*}, \alpha_{\lambda}^{*}\right)=(3 / 16,0), \\
(\mathrm{B}):\left(\alpha_{\mathrm{SC}}^{*}, \alpha_{\lambda}^{*}\right)=(27 / 128,3 / 16),
\end{gathered}
$$

which are marked in Fig. 1. The RG flows are also shown in Fig. 1. It is clearly seen that the fixed point (B) is indeed IR attractive. Now we suppose that the theory is given near the fixed point (A) and comes close to the IR attractive fixed point (B) at scale $\Lambda_{\mathrm{SC}}$ that is not much larger than the electro-weak scale, say $10 \sim 100 \mathrm{TeV}$ [13].

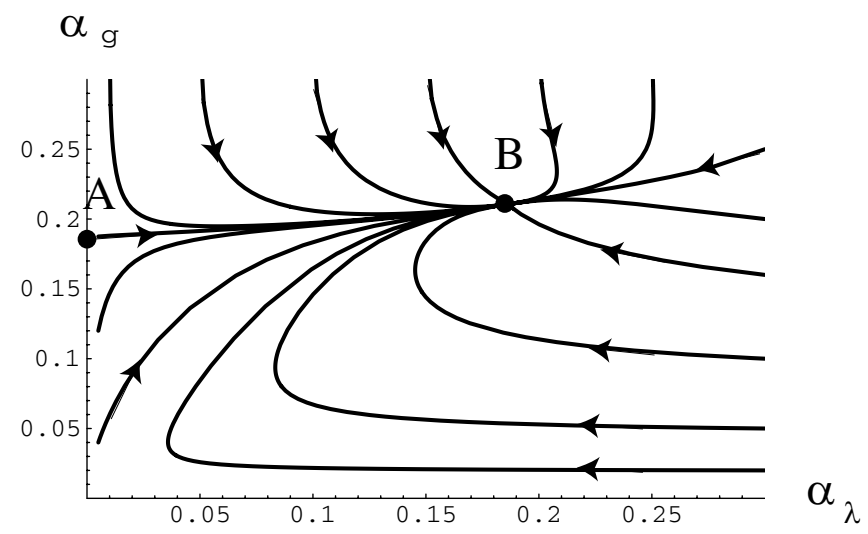

FIG. 1. The RG flows obtained by solving Eqs. (11) and (12) are shown. The arrows indicate the direction toward IR. The points A and B represent the fixed points (13) and (14) respectively.
There are other relevant operators than $\lambda \Phi \bar{\phi} H_{u}$ at the fixed point (A). We include these operators into the superpotential and suppose that it is given at the IR attractive fixed point as

$$
\begin{aligned}
W= & Y_{t} Q_{3} \bar{u}_{3} H_{u}+\lambda_{*} \Phi \bar{\phi} H_{u}+\kappa_{*} Q_{3} \Omega \bar{\Phi}+\kappa_{*}^{\prime} \phi \bar{\Omega} \bar{u}_{3} \\
& +\mu H_{u} H_{d}+M_{\Phi} \Phi \bar{\Phi}+M_{\phi} \phi \bar{\phi}+M_{\Omega} \Omega \bar{\Omega} .
\end{aligned}
$$

Here the supersymmetric mass terms are also introduced. We do not consider the Yukawa coupling with $H_{d}$ for simplicity. When we neglect the gauge couplings $g_{3}^{\prime}$ as well as $g_{2}$ and $g_{1}$, the existence of the IR attractive fixed point with large Yukawa couplings $\lambda_{*}, \kappa_{*}, \kappa_{*}^{\prime}$ may be shown by using the anomalous dimensions approximated in the one-loop level. Then we may find an IR attractive fixed point and the explicit couplings are given by $\left(\alpha_{\mathrm{SC}}^{*}, \alpha_{\lambda}^{*}, \alpha_{\kappa}^{*}, \alpha_{\kappa^{\prime}}^{*}\right) \simeq(0.38,0.34,0.25,0.29)$, where $\alpha_{\kappa}=$ $|\kappa|^{2} / 8 \pi^{2}$. Of course such a perturbative analysis is not so trustworthy for these large couplings, though it may offer us an indication of the fixed point.

The anomalous dimensions for the extra matter fields are modified in the presence of these Yukawa terms. Then the anomalous dimension of Higgs is given by

$$
\gamma_{H_{u}}=-\left(\gamma_{\Phi}+\gamma_{\phi}\right)
$$

which is expected to be about one. The mass parameter $\mu$ is also suppressed as

$$
\mu(\Lambda)=\left(\frac{\Lambda}{\Lambda_{\mathrm{SC}}}\right)^{\gamma_{H_{u}}} \mu\left(\Lambda_{\mathrm{SC}}\right),
$$

while the mass parameters $M_{\Phi}, M_{\phi}, M_{\Omega}$ are enhanced. Therefore the decoupling scale of the superconformal sector is much larger than $\mu$. In this model, not only Higgs but also quarks acquire positive anomalous dimensions, therefore the Yukawa couplings $Y_{t}$ is suppressed rather strongly.

The spontaneous breakdown of the gauge symmetry $S U(3)_{\mathrm{SC}} \times S U(3)_{C}^{\prime}$ to the diagonal subgroup $S U(3)_{C}$ occurs, if the bifundamental matter fields acquire vacuum expectation values (VEVs) as

$$
\left\langle\Omega_{a}^{A}\right\rangle=\omega \delta_{a}^{A}, \quad\left\langle\bar{\Omega}_{A}^{a}\right\rangle=\bar{\omega} \delta_{A}^{a},
$$

where $A, a=1,2,3$ are indices of the fundamental representations. These VEVs also bring about mixing between the quark superfields and the superconformal matter fields simultaneously. By substituting these VEVs into the superpotential, the mass terms are modified effectively as

$$
W \sim M_{\Phi}\left(\Phi+\frac{\kappa_{*} \omega}{M_{\Phi}} Q_{3}\right) \bar{\Phi}+M_{\phi} \phi\left(\bar{\phi}+\frac{\kappa_{*}^{\prime} \bar{\omega}}{M_{\phi}} \bar{u}_{3}\right) .
$$

These mass terms induce mixing between $S U(2)_{W}$ doublets, $Q_{3}$ and $\Phi^{\prime}$, and also singlets, $\bar{u}_{3}$ and $\bar{\phi}$. The mass eigenmodes $\left(Q_{3}^{\prime}, \Phi^{\prime}\right)$ and $\left(\bar{u}_{3}^{\prime}, \bar{\phi}^{\prime}\right)$ are given through rotations, 


$$
\begin{aligned}
& \left(\begin{array}{l}
Q_{3}^{\prime} \\
\Phi^{\prime}
\end{array}\right)=\left(\begin{array}{cc}
\cos \theta_{L} & -\sin \theta_{L} \\
\sin \theta_{L} & \cos \theta_{L}
\end{array}\right)\left(\begin{array}{c}
Q_{3} \\
\Phi
\end{array}\right), \\
& \left(\begin{array}{c}
\bar{u}_{3}^{\prime} \\
\bar{\phi}^{\prime}
\end{array}\right)=\left(\begin{array}{cc}
\cos \theta_{R} & -\sin \theta_{R} \\
\sin \theta_{R} & \cos \theta_{R}
\end{array}\right)\left(\begin{array}{c}
\bar{u}_{3} \\
\bar{\phi}
\end{array}\right),
\end{aligned}
$$

where $\tan \theta_{L}=\kappa_{*} \omega / M_{\Phi}$ and $\tan \theta_{R}=\kappa_{*}^{\prime} \bar{\omega} / M_{\phi}$. The massless modes $Q_{3}^{\prime}$ and $\bar{u}_{3}^{\prime}$ are regarded as the third generation quark fields.

After decoupling of $\Omega$ and $\bar{\Omega}$, the superpotential is reduced to be

$$
\begin{aligned}
W= & \lambda_{*}\left(\cos \theta_{L} \Phi^{\prime}-\sin \theta_{L} Q_{3}^{\prime}\right)\left(\cos \theta_{R} \bar{\phi}^{\prime}-\sin \theta_{R} \bar{u}_{3}^{\prime}\right) H_{u} \\
& +M_{\Phi}^{\prime} \Phi^{\prime} \bar{\Phi}+M_{\phi}^{\prime} \phi \bar{\phi}^{\prime}+\mu H_{u} H_{d},
\end{aligned}
$$

where $M_{\Phi}^{\prime}=M_{\Phi} / \cos \theta_{L}$ and $M_{\phi}^{\prime}=M_{\phi} / \cos \theta_{R}$. We have omitted the original top-Yukawa coupling $Y_{t}$, since it is suppressed. However a new top-Yukawa term is induced in this effective superpotential. The effective top-Yukawa coupling is given by

$$
Y_{t}^{\text {eff }}=\lambda_{*} \sin \theta_{L} \sin \theta_{R} .
$$

The Higgs field VEV $\left\langle H_{u}\right\rangle=v_{u}=v \sin \beta$, which causes the EW symmetry breaking, generates a mass matrix among top quarks and the extra heavy "quarks". It is seen that top quark is mixed with the heavy quarks furthermore, but this mixing is very small due to large weak symmetric masses $M_{\Phi}^{\prime}$ and $M_{\phi}^{\prime}$. The mass of top quark is simply given by $m_{t}=Y_{t}^{\text {eff }} v_{u}$. Therefore the coupling $Y_{t}^{\text {eff }}$ should be about unity for the realistic value of $m_{t}$. This is realized when $\sin \theta_{L} \sim \sin \theta_{R} \sim 1 / \sqrt{\lambda_{*}}$. The fixed point couplings $\lambda_{*}, \kappa_{*}$ may be roughly estimated to be $2 \pi / 3$. Thus we find that the large top quark mass is generated, if the VEV $\omega$ is also given to be mass scale of the extra matter fields. It is also noted that bottom quarks are not mixed with the heavy fields. Therefore there is no tree-level corrections to the Z-boson decay width $R_{b}=\Gamma[Z \rightarrow$ $b \bar{b}] / \Gamma[Z \rightarrow$ hadrons $]$. Consistency with the precision tests of the EW theory is discussed in the last part of section IV.

Next we consider the soft scalar mass of Higgs obtained at the decoupling scale from the superconformal sector. The exact beta functions [19] and the spurion method [19$24,26]$ enable us to show that the gaugino mass and the Aparameters in a superconformal gauge theory decrease in proportion to certain powers of the scale towards IR $[14,15]$. Therefore these supersymmetry breaking parameters are almost vanishing at the scale of $\Lambda_{\mathrm{SC}}$, where the Yukawa coupling $\lambda$ reaches near the IR fixed point. Since the coupling $Y_{t}$ also becomes negligible below the scale of $\Lambda_{\mathrm{SC}}$, the RG equations for $m_{\Phi}^{2}, m_{\bar{\phi}}^{2}, m_{H_{u}}^{2}$ are just identical to Eqs. (4) at one-loop level. It is sufficient to replace $m_{Q_{3}}^{2}, m_{\bar{u}_{3}}^{2}$ to $m_{\Phi}^{2}, m_{\bar{\phi}}^{2}$ and $Y_{t}, M_{3}, A_{t}$ to $\lambda, M_{\mathrm{SC}}, A_{\lambda}$ respectively in Eqs. (4). Here, however, the soft breaking parameters $M_{\mathrm{SC}}, A_{\lambda}$ have been sufficiently suppressed already at $\Lambda_{\mathrm{SC}}$. Therefore these soft masses, especially $m_{H_{u}}^{2}$, are not enhanced below $\Lambda_{\mathrm{SC}}$, as is discussed in the previous section. Consequently if the scalar masses $m_{\Phi}^{2}, m_{\bar{\phi}}^{2}, m_{H_{u}}^{2}$ are all small at scale of $\Lambda_{\mathrm{SC}}$, then $m_{H_{u}}^{2}$ becomes also small at low energy.

Now we suppose that the theory stays near the fixed point (A) given in Eq. (13) above a certain scale of $\Lambda_{\mathrm{SC}}^{\prime}>$ $\Lambda_{\mathrm{SC}}$, and the coupling $\lambda$ is tiny. There the Higgs field is separated from the strongly interacting sector completely. In the absence of the large top-Yukawa coupling $Y_{t}$, the dominant radiative corrections to the Higgs mass parameters are given by the $S U(2)_{W}$ gauge interaction, which are irrelevant to fine-tuning. On the other hand only the gauge interaction is strong in the superconformal sector and the extra vectorlike matter fields are subject to the same corrections. Then, if the scalar masses of these fields are given to be universal at the fundamental scale, the scalar masses are reduced through the superconformal dynamics $[12,14,17]$. Thus the scalar masses $m_{\Phi}^{2}$ and $m_{\bar{\phi}}^{2}$ as well as the gaugino mass $M_{\mathrm{SC}}$ have been suppressed at the scale $\Lambda_{\mathrm{SC}}^{\prime}$.

Finally let us consider the RG behavior of the soft scalar masses at the transition region from fixed point (A) to (B) in more details. It is noted that the A-parameter $A_{\lambda}$ is not suppressed before approaching the fixed point (B). Meanwhile the Yukawa coupling $\lambda$ grows rapidly and exceed $\mathrm{O}(1)$ quickly. Therefore we may wonder that the A-parameter affects RG behavior of the soft scalar masses significantly.

In order to see this, we solve the coupled RG equations for $m_{\Phi}^{2}, m_{\bar{\phi}}^{2}, m_{H_{u}}^{2}$ and $A_{\lambda}$. The explicit equations may be written down immediately by applying 1-loop anomalous dimensions to the exact formulas [19-24,26]. First the RG equation for $A_{\lambda}$ is found to be

$$
\frac{d A_{\lambda}}{d \ln \mu}=\frac{16}{3} \alpha_{\mathrm{SC}} M_{\mathrm{SC}}+6 \alpha_{\lambda} A_{\lambda},
$$

where $\alpha_{\mathrm{SC}}=g_{\mathrm{SC}}^{2} / 8 \pi^{2}$ and $\alpha_{\lambda}=|\lambda|^{2} / 8 \pi^{2}$ again. It is seen that the A-parameter $A_{\lambda}$ is suppressed rapidly as $\lambda$ grows in the absence of the gaugino mass $M_{\mathrm{SC}}$. The RG equations for the scalar masses may be obtained similarly and are found to be

$$
\begin{gathered}
\frac{d m_{\Phi}^{2}}{d \ln \mu}=-\frac{8}{3} \alpha_{\mathrm{SC}}\left(2\left|M_{\mathrm{SC}}\right|^{2}+\Delta_{g}\right)+\alpha_{\lambda}\left(\left|A_{\lambda}\right|^{2}+\Sigma\right), \\
\frac{d m_{\bar{\phi}}^{2}}{d \ln \mu}=-\frac{8}{3} \alpha_{\mathrm{SC}}\left(2\left|M_{\mathrm{SC}}\right|^{2}+\Delta_{g}\right)+2 \alpha_{\lambda}\left(\left|A_{\lambda}\right|^{2}+\Sigma\right),
\end{gathered}
$$

$$
\frac{d m_{H_{u}}^{2}}{d \ln \mu}=3 \alpha_{\lambda}\left(\left|A_{\lambda}\right|^{2}+\Sigma\right),
$$

where $\Delta_{g}$ and $\Sigma$ are given in terms of the scalar masses as 
INDUCED TOP-YUKAWA COUPLING AND SUPPRESSED...

$$
\begin{gathered}
\Delta_{g}=\frac{\alpha_{\mathrm{SC}}}{1-3 \alpha_{\mathrm{SC}}}\left(3\left|M_{\mathrm{SC}}\right|^{2}-m_{\Phi}^{2}-\frac{1}{2} m_{\bar{\phi}}^{2}\right), \\
\Sigma=m_{\Phi}^{2}+m_{\bar{\phi}}^{2}+m_{H_{u}}^{2} .
\end{gathered}
$$

Here we neglected dependence on other scalar masses in $\Delta_{g}$ since these are suppressed.

In Fig. 2 and 3 an example of the solutions to Eqs. (24)(27) coupled with Eqs. (11) and (12) running aspect of $A_{\lambda}$, is shown in the transition between the fixed points. Indeed the A-parameter is suppressed rapidly as the Yukawa coupling $\alpha_{\lambda}$ approaches the IR fixed point (B). The solutions for scalar masses $m_{\Phi}^{2}, m_{\bar{\phi}}^{2}, m_{H_{u}}^{2}$ are shown in Fig. 3. We find that $m_{H_{u}}^{2}$ is reduced somewhat by the A-parameter, but the correction is not considerable, even though $A_{\lambda}$ is relatively large at $\Lambda_{\mathrm{SC}}^{\prime}$.

So far we have not taken into account the $S U(3)_{C}^{\prime}$ effects to the soft scalar masses and have assumed that all scalar masses of the matter fields charged under $S U(3)_{\mathrm{SC}}$ are suppressed by superconformal dynamics [14]. However the extra matter fields $\Omega$ and $\bar{\Omega}$, whose VEVs bring about the symmetry breaking and the mixing between the original top quark and superconformal matter fields, are charged under $S U(3)_{C}^{\prime}$. So one may wonder if $m_{\Omega}^{2}$ and $m_{\bar{\Omega}}^{2}$ are enhanced by the $S U(3)_{C}^{\prime}$ correction just like squark masses, since mass of the $S U(3)_{C}^{\prime}$ gaugino is supposed to become large at low energy. In addition, $m_{\Phi}^{2}$ and $m_{\bar{\phi}}^{2}$ are not just suppressed but are also enhanced as much as $m_{\Omega}^{2}$, because these scalar masses should satisfy the IR sum rule [15].

This may be avoided as follows. It is noted that the symmetry allows interaction among $\Omega$ given by

$$
\Delta W=\eta_{*} \epsilon_{A B C} \epsilon^{a b c} \Omega_{a}^{A} \Omega_{b}^{B} \Omega_{c}^{C},
$$

in the superpotential. Then the IR sum rule tells us $m_{\Omega}^{2} \rightarrow 0$

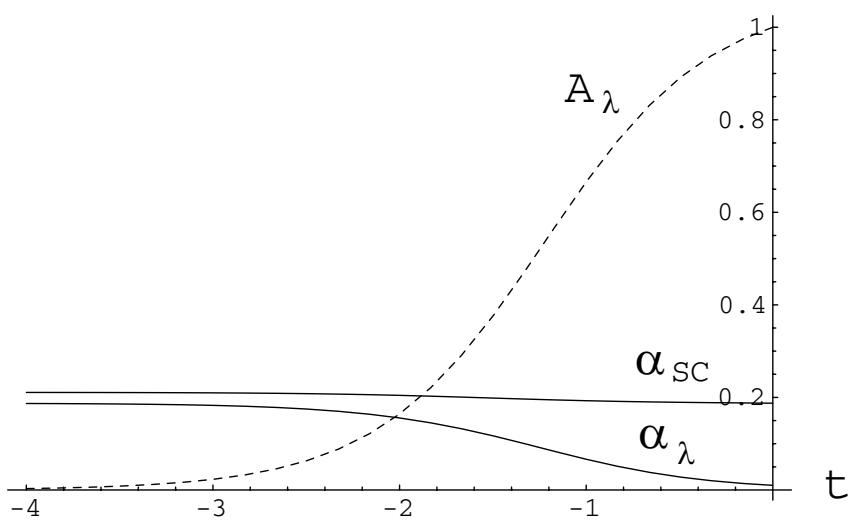

FIG. 2. Running behavior of $A_{\lambda}$ as well as the couplings, $\alpha_{\mathrm{SC}}=g_{\mathrm{SC}}^{2} / 8 \pi^{2}$ and $\alpha_{\lambda}=|\lambda|^{2} / 8 \pi^{2}$, in the transition region between the fixed points (A) and (B) is shown. The renormalization scale $\mu$ is represented by $t=\log _{10}\left(\mu / \Lambda_{\mathrm{SC}}^{\prime}\right)$. These lines are obtained by solving Eqs. (11), (12), and (24).

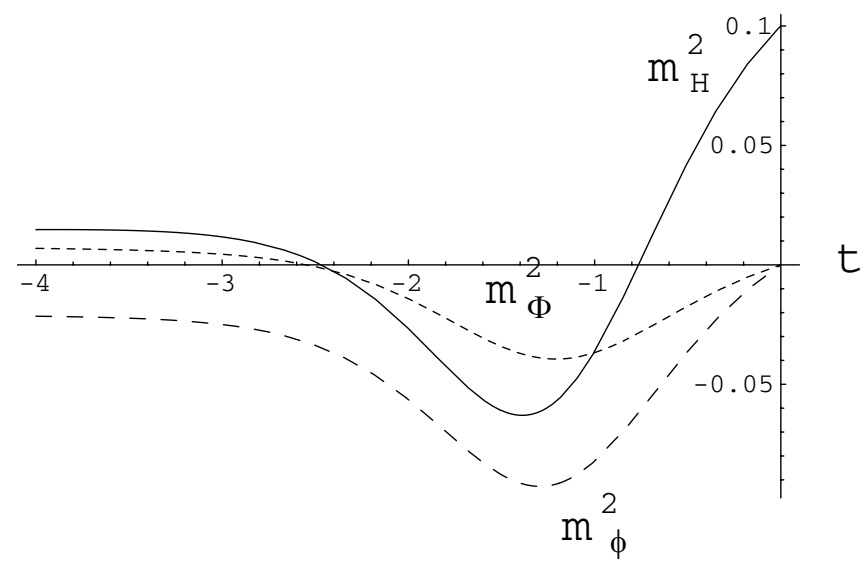

FIG. 3. Running behavior of soft scalar masses $m_{H}^{2}, m_{\Phi}^{2}$ and $m_{\bar{\phi}}^{2}$ is shown. The renormalization scale $\mu$ is represented by $t=$ $\log _{10}\left(\mu / \Lambda_{\mathrm{SC}}^{\prime}\right)$. We set $m_{H_{u}}^{2} / A_{\lambda}^{2}=0.1$ at $t=0$ as an example. These lines are obtained by solving Eqs. (24)-(27) coupled with Eqs. (11) and (12).

at low energy. In practice, $m_{\Omega}^{2}$ does not vanish because of the $S U(3)_{C}^{\prime}$ corrections. However we note that the logarithmic correction does disappear [13]. This may be demonstrated by using the one-loop RG equation for $m_{\Omega}^{2}$, which is given by

$$
16 \pi^{2} \frac{d m_{\Omega}^{2}}{d \ln \mu}=4\left|\eta_{*}\right|^{2} m_{\Omega}^{2}-\frac{32}{3} g_{3}^{2}\left|M_{3}\right|^{2} .
$$

If we use $\left|\eta_{*}\right| \sim 4 \pi$ by naive dimensional analysis, then the soft mass converges as

$$
m_{\Omega}^{2} \rightarrow \frac{1}{6 \pi^{2}} g_{3}^{2}\left|M_{3}\right|^{2}
$$

Thus $m_{\Omega}^{2}$ becomes small enough at the scale of $\Lambda_{\mathrm{SC}}^{\prime}$. Then the IR sum rule guarantees that the soft scalar masses $\left|m_{\Phi}^{2}\right|$ and $\left|m_{\bar{\phi}}^{2}\right|$ are as small as $m_{\Omega}^{2}$. Thus although the mass of $S U(3)_{C}^{\prime}$ gaugino may be large, the soft scalar masses in the superconformal sector can be suppressed.

\section{SOME PHENOMENOLOGICAL ASPECTS}

First we consider the gauge coupling unification. Introduction of the extra matter fields charged under the SM gauge group alters the gauge beta functions. As was mentioned before, the extra matter fields are not combined into representations of $S U(5)$. Therefore the Weinberg angle is shifted from the value obtained in the MSSM, which fits in the experimental data.

The running gauge couplings $\alpha_{a}=g_{a}^{2} / 4 \pi(a=1,2,3)$ for the SM gauge groups are given explicitly at one-loop level; 


$$
\begin{aligned}
\alpha_{a}^{-1}(\mu)= & \alpha_{a}^{-1}\left(M_{Z}\right)+\frac{b_{a}^{(\text {low })}}{2 \pi} \ln \frac{M_{\Phi}}{M_{Z}}+\frac{b_{a}^{(\mathrm{SC})}}{2 \pi} \ln \frac{\Lambda_{\mathrm{SC}}}{M_{\Phi}} \\
& +\frac{b_{a}^{(\text {high })}}{2 \pi} \ln \frac{\mu}{\Lambda_{\mathrm{SC}}}
\end{aligned}
$$

where $M_{\Phi}$ stands for the decoupling scale of the extra matter fields. We also suppose the scale $\Lambda_{\mathrm{SC}}^{\prime}$ to be rather close to $\Lambda_{\mathrm{SC}}$ and do not consider running between these scales. To be explicit we use $M_{\Phi}=1 \mathrm{TeV}$ and $\Lambda_{\mathrm{SC}}=$ $10 \mathrm{TeV}$ in the calculations below.

Now we calculate the beta function coefficients $b_{a}$ in each energy region. In the present model only the Higgsino and probably the right-handed sleptons are supposed to be light among superpartners of the SM fields. Then $b_{a}^{\text {(low) }}$ may be evaluated as

$$
\left(b_{3}^{\text {(low) }}, b_{2}^{\text {(low) }}, b_{1}^{(\text {low) }}\right)=(-7,-15 / 6,51 / 10) .
$$

Though the running below $M_{\Phi}$ is rather model dependent and uncertain, the contribution is not important anyway.

Next we consider the region where the MSSM coupled with the superconformal sector. We suppose that not only the coupling $\lambda$ but also $\kappa$ and $\kappa^{\prime}$ are reaching their IR fixed points below $\Lambda_{\mathrm{SC}}$ in the superpotential given by (15). There the anomalous dimensions of the fields charged under $S U(3)_{\mathrm{SC}}, \gamma_{\Phi}, \gamma_{\bar{\Phi}}$ and so on, are $-1 / 2$ approximately. Meanwhile $\gamma_{H_{u}}, \gamma_{Q_{3}}$ and $\gamma_{\bar{u}_{3}}$ may be evaluated to be about one. The gauge beta function coefficients are given in general by $b_{a}=-b_{a}^{(\mathrm{MSSM})}+\sum_{i}(1 / 2)\left(1-\gamma_{\phi_{i}}\right)$, where the chiral fields $\phi_{i}$ belong to the fundamental representation. Therefore we may evaluate $b_{a}^{(\mathrm{SC})}$ as

$$
\left(b_{3}^{(\mathrm{SC})}, b_{2}^{(\mathrm{SC})}, b_{1}^{(\mathrm{SC})}\right) \sim(0,7 / 2,81 / 10) .
$$

Above the scale $\Lambda_{\mathrm{SC}}$ (or $\Lambda_{\mathrm{SC}}^{\prime}$ ), the couplings of the superconformal sector are also supposed to be small. Therefore the coefficients may be evaluated by one-loop approximation and are found to be

$$
\left(b_{3}^{(\text {high })}, b_{2}^{(\text {high })}, b_{1}^{(\text {high) }}\right)=(0,4,42 / 5) .
$$

By using these coefficients in the gauge couplings Eqs. (34), we may examine the aspect of their unification. For example it is seen that $\alpha_{3}$ and $\alpha_{1}$ cross each other at the scale about $10^{18} \mathrm{GeV}$. It may be interesting that this is so high as the Planck scale or the string scale. If we assume that $\alpha_{2}$ coincides with other gauge couplings at this scale, then the Weinberg angle is found to be $\sin ^{2} \theta_{W} \approx 0.24$, which is still very close to the realistic value.

Next we also examine low energy spectrum of the superparticles in this model. Specifically we consider the mSUGRA scenario with the universal gaugino mass $M_{1 / 2}$ and the universal scalar mass $m_{0}$, which is now assumed to be very small at the Planck scale. The running behavior of the gauge couplings are rather different from that in the MSSM. Therefore the soft parameters obtained at low energy are distinct from those in the ordinary mSUGRA scenario.

It will be enough to take account of the corrections above the scale of $\Lambda_{\mathrm{SC}}$ at the one-loop level. Since the gaugino masses satisfy the relation, $M_{3}(\mu): M_{2}(\mu): M_{1}(\mu)=\alpha_{3}(\mu): \alpha_{2}(\mu): \alpha_{1}(\mu)$, their ratios are the same as in the mSUGRA case;

$$
M_{2} \approx 0.29 M_{3}, \quad M_{1} \approx 0.14 M_{3} .
$$

Contrary to this, the squark masses are considerably enhanced by the $S U(3)_{C}$ corrections, since $\alpha_{3}$ is not reduced at higher energy scale. Explicitly the correction may be given as

$$
\Delta m_{\tilde{q}}^{2}=\frac{32}{12 \pi} \alpha_{3}\left|M_{3}\right|^{2} \ln \frac{M_{\mathrm{Pl}}}{\Lambda_{\mathrm{SC}}},
$$

from which the squark masses at low energy are expected to be

$$
m_{\tilde{Q}} \sim m_{\tilde{q}} \sim 1.6 M_{3} .
$$

Similarly we may obtain other soft mass parameters as

$$
m_{\tilde{L}} \sim 0.5 M_{3}, \quad m_{H_{d}} \sim 0.5 M_{3}, \quad m_{\tilde{e}} \sim 0.28 M_{3} .
$$

We note that the soft masses in this model are relatively heavier than those in the conventional scenarios. In the presence of $m_{0}$ at the Planck scale, these are raised up more.

On top of that, the most characteristic feature of our model is that Higgs and Higgsino fields are much lighter than squark and sleptons. The soft scalar mass $m_{H_{u}}^{2}$ receives radiative corrections only below the decoupling scale $M_{\Phi}$ and is expected to be one order smaller than $m_{\tilde{t}}^{2}$. The $\mu$-parameter is also suppressed. This spectrum is favorable in the following respects. One is the neutralino relic abundance [26], which has been constrained precisely by WMAP. In the most parameter region of mSUGRA the lightest neutralino is Bino-like, and the relic abundance constraint leads to stringent upper bound on scalar and/or gaugino masses. In our model the lightest supersymmetric particle (LSP) is given as a gaugino-Higgsino mixture or even as a Higgsino dominant component. It has been known that the relic abundance is well explained in the case of such spectra, which is similar to the focus point region $[17,26]$.

Another advantageous feature is on the stability of the MSSM scalar potential. The mass parameters in the MSSM must satisfy constraints against charge and/or color breaking and unbounded-from-below. The most serious unbounded-from-below direction of the MSSM scalar potential involves the Higgs and slepton fields, i.e. the socalled UFB-3 direction [27]. If the quantity $m_{H_{u}}^{2}+m_{\tilde{L}}^{2}$ turns out to be negative, then the potential becomes unstable along the UFB-3 direction. In our scenario, the Higgs soft mass is suppressed, and therefore the UFB-3 bound can be relaxed. 
Lastly we also consider constraints by the precision measurements of the EW theory. In our scenario it is essential to incorporate the mixing of the top quark with a heavy extra field in order to induce a large top-Yukawa coupling. After symmetry breaking of $S U(3)_{\mathrm{SC}} \times$ $S U(3)_{C}^{\prime} \rightarrow S U(3)_{C}$, the extra fields $\left(\Phi^{\prime}, \bar{\Phi}\right)$ and $\left(\phi, \bar{\phi}^{\prime}\right)$ follow the effective superpotential (22). It should be noted that these extra fields are vectorlike and have large proper masses. We come across a rather similar situation in the socalled top quark seesaw models [28]. In these models, a vectorlike pair of weak singlet fermions $\left(\chi_{L}, \chi_{R}\right)$ are introduced and mixed mass terms with top quarks as well as mass of themselves are generated dynamically.

In general, mixing with extra quarks causes large oblique corrections. However contributions of the vectorlike quarks are suppressed due to decoupling with the large masses allowed by the EW symmetry. Therefore the isospin breaking parameter $\delta \rho$ or the T-parameter is found to be small enough, when the mass for $\left(\chi_{L}, \chi_{R}\right)$ is as heavy as several $\mathrm{TeV}$. The $\mathrm{S}$-parameter is suppressed more than the T-parameter. After the EWSB, similar mixed mass terms with extra matter fields appear in our model as well, though not only weak singlets but also weak doublets are incorporated there. Therefore the oblique corrections may be also suppressed sufficiently, as long as the decoupling scale $M_{\Phi}$ and $M_{\phi}$ are as large as $10 \mathrm{TeV}{ }^{3}$ Another constraint is put by the Z-boson decay width $R_{b}=\Gamma[Z \rightarrow b \bar{b}] / \Gamma[Z \rightarrow$ hadrons], which gives also severe lower bounds for the explicit mass of $\left(\chi_{L}, \chi_{R}\right)$ in the top quark seesaw models [28]. In our models, however, the bottom quark does not have mixing with the extra quarks, and therefore there is no tree-level contributions for $R_{b}$. The corrections appear only through loop diagrams, which are found to be much smaller than the experimental bound, $\delta R_{b}<10^{-3}$.

\section{CONCLUSION AND DISCUSSION}

In this article we considered a scenario ameliorating the supersymmetric little hierarchy problem in the MSSM. We concentrated particularly on models with heavy gluinos and squarks as given in mSUGRA and GMSB scenarios. In order to suppress radiative corrections to Higgs mass parameters, we assumed that top-Yukawa coupling is small above TeV. The large top quark mass is effectively generated through top quark mixing with strongly coupled sector around $\mathrm{TeV}$ scale. Accordingly, the Higgs field has a large Yukawa coupling with the extra matter fields instead of the top quark. Nevertheless, corrections to the soft scalar mass of Higgs can be made suppressed.

To be explicit, we presented a model in which the Higgs field couples with a superconformal gauge theory. Then it

\footnotetext{
${ }^{3}$ In this rough estimation, we consider corrections only by the fermionic fields without taking supersymmetry into consideration. Explicit evaluation of the oblique corrections in our model is leave for future study.
}

is shown that Higgs is sequestered from large supersymmetry breaking effects and, therefore, the soft scalar mass of Higgs does not receive a large radiative correction. Thus the fine-tuning problem is ameliorated. Moreover the large anomalous dimension of the Higgs field suppresses the $\mu$-term. Thus Higgsino also appears light compared with squarks and sleptons, which is favored for the neutralino dark matter scenario. The GMSB models with messengers that are neutral under the strong gauge group is also considerable and is supposed to present a similar sparticle spectrum. This will be reported elsewhere.

In the model building we just assumed the symmetry breaking of $S U(3)_{\mathrm{SC}} \times S U(3)_{C}^{\prime}$ to occur also around $\mathrm{TeV}$ scale. Though it is interesting to construct an explicit model that implements this symmetry breaking, we leave it for future study. We also assumed that only top quarks acquire mixing terms with the extra matter fields. However this may be related to the question why only top quark mass is prominently large. In this respect it may be interesting to extend the model to incorporate three generations of quarks, and to consider the quark mass matrix.

Lastly some comments on the other corrections induced by the extra matter fields are in order. Recently Babu et. al. [10] considered similar models to ours, though they were not concerned with soft scalar masses and their models are rather weakly coupled. They examined the cases that additional contributions to the quartic coupling of Higgs bosons in the MSSM are induced by the loop effect of extra matter fields coupled to Higgs fields. Then the mass of the lightest neutral Higgs boson may be raised up considerably. In our model the same kind of correction also exists and is given roughly by

$$
\Delta m_{h^{0}}^{2} \sim \frac{3}{8 \pi^{2}}\left(-m_{Z}^{2} \cos ^{2} 2 \beta \lambda_{*}^{2}+2 v^{2} \sin ^{4} \beta \lambda_{*}^{4}\right) t_{1},
$$

where $t_{1}=\ln \left(1+m_{\Phi}^{2} / M_{\Phi}^{2}\right)$ and we used $M_{\bar{\phi}}=M_{\Phi}$ and $m_{\bar{\phi}}^{2}=m_{\Phi}^{2}$ for simplicity. However the supersymmetric mass $M_{\Phi}$ is now taken to be fairly large, while the soft mass $m_{\Phi}$ is suppressed. Therefore enhancement of the lightest Higgs boson mass is found to be very small in the present model.

We add remarks on the decoupling effects to the soft supersymmetry breaking parameters. We note that Bparameters accompanied with the mass terms of the extra matter fields exist and are not suppressed by the superconformal dynamics. Even the B-parameters are induced through corrections with the gaugino for $S U(3)_{\mathrm{SC}}$, since mass of the gaugino is not small at very high energy scale. Then soft supersymmetry breaking parameters in the MSSM receive threshold corrections due to the Bparameters when the extra matter fields decouple at the mass scale of $M_{\Phi}$. For gaugino masses, the threshold corrections are similar to the gauge mediation effect. What we especially concern ourselves about is the effect to the soft scalar mass of Higgs, $m_{H_{u}}^{2}$. We can evaluate such 
threshold corrections in a manner outlined in Ref. [29]. Then we find that the threshold correction to $m_{H_{u}}^{2}$ may be evaluated as

$$
\Delta m_{H_{u}}^{2} \sim-|B|^{2} \Delta\left(\frac{d \gamma_{H_{u}}}{d \ln \mu}\right),
$$

where $\Delta(*)$ represents the difference generated through the decoupling. Here the scale dependence of the anomalous dimension of the Higgs field is brought about by the SM gauge interactions in the leading order. Therefore, the threshold corrections to $m_{H_{u}}^{2}$ are found to be insignificant for the present problem.

\section{ACKNOWLEDGMENTS}

T. K. is supported in part by the Grants-in-Aid for Scientific Research (No. 16028211) and the Grant-in-Aid for the 21st Century COE "The Center for Diversity and Universality in Physics" from the Ministry of Education, Science, Sports and Culture, Japan. H.N. and H. T. are supported in part by the Grants-in-Aid for Scientific Research (No. 16540238 and No. 13135210, respectively) from the Ministry of Education, Science, Sports and Culture, Japan.
[1] R. Barbieri and G.F. Giudice, Nucl. Phys. B306, 63 (1988).

[2] P. H. Chankowski, J. R. Ellis, and S. Pokorski, Phys. Lett. B 423, 327 (1998); Nucl. Phys. B544, 39 (1999); G. L. Kane and S. F. King, Phys. Lett. B 451, 113 (1999); M. Bastero-Gil, G. L. Kane, and S. F. King, Phys. Lett. B 474, 103 (2000).

[3] K. Inoue, A. Kakuto, H. Komatsu, and S. Takeshita, Prog. Theor. Phys. 67, 1889 (1982); Prog. Theor. Phys. 68, 927 (1982); 70, 330E (1983); Prog. Theor. Phys. 71, 413 (1984); L. E. Ibanez and G. G. Ross, Phys. Lett. B 110, 215 (1982); L. Alvarez-Gaume, M. Claudson, and M. B. Wise, Nucl. Phys. B207, 96 (1982).

[4] G. Abbiendi et al., ALEPH, DELPHI, L3 and OPAL Collaborations, (The LEP Working Group for Higgs Boson Searches), Phys. Lett. B 565, 61 (2003).

[5] Y. Okada, M. Yamaguchi, and T. Yanagida, Phys. Lett. B 262, 54 (1991); H. E. Haber and R. Hempfling, Phys. Rev. Lett. 66, 1815 (1991); J. R. Ellis, G. Ridolfi, and F. Zwirner, Phys. Lett. B 262, 477 (1991).

[6] K. Agashe and M. Graesser, Nucl. Phys. B507, 3 (1997).

[7] J. A. Casas, J. R. Espinosa, and I. Hidalgo, J. High Energy Phys. 01 (2004) 008.

[8] R. Harnik, G. D. Kribs, D. T. Larson, and H. Murayama, Phys. Rev. D 70, 015002 (2004); S. Chang, C. Kalic, and R. Muhbubani, Phys. Rev. D 71, 015003 (2005); A. Birkedal, Z. Chacko, and Y. Nomura, Phys. Rev. D 71, 015006 (2005).

[9] P. Batra, A. Delgado, D.E. Kaplan. and T. M.P. Tait, J. High Energy Phys. 02 (2004) 043; J. High Energy Phys. 06 (2004) 032.

[10] K. S. Babu, I. Gogoladze, and C. Kolda, hep-ph/0410085.

[11] P. J. Fox, A. E. Nelson, and N. Weiner, J. High Energy Phys. 08 (2002) 035;Z. Chacko, P. J. Fox, and H. Murayama, Nucl. Phys. B706, 53 (2005).

[12] A. Birkedal, Z. Chacko, and M. K. Gaillard, J. High Energy Phys. 10 (2004) 036.

[13] T. Kobayashi and H. Terao, J. High Energy Phys. 07 (2004) 026.

[14] A. Karch, T. Kobayashi, J. Kubo, and G. Zoupanos, Phys. Lett. B 441, 235 (1998); M. A. Luty and R. Rattazzi, J. High Energy Phys. 11 (1999) 001.
[15] A.E. Nelson and M. J. Strassler, J. High Energy Phys. 09 (2000) 030; J. High Energy Phys. 07 (2002) 021; T. Kobayashi and H. Terao, Phys. Rev. D 64, 075003 (2001); T. Kobayashi, H. Nakano, and H. Terao, Phys. Rev. D 65, 015006 (2002); T. Kobayashi, H. Nakano, T. Noguchi, and H. Terao, Phys. Rev. D 66, 095011 (2002).

[16] M. A. Luty and T. Okui, hep-ph/0409274.

[17] J. L. Feng, K. T. Matchev, and T. Moroi, Phys. Rev. Lett. 84, 2322 (2000); Phys. Rev. D 61, 075005 (2000); J. L. Feng, K. T. Matchev, and F. Wilczek, Phys. Lett. B 482, 388 (2000).

[18] N. Seiberg, Nucl. Phys. B435, 129 (1995); K. Intrilligator and N. Seiberg, Nucl. Phys. B Proc. Suppl. 45, 1 (1996).

[19] V. Novikov, M. Shifman, A. Vainstein, and V. Zakharov, Nucl. Phys. B229, 381 (1983); Phys. Lett. B 166, 329 (1986); M. Shifman, Int. J. Mod. Phys. A 11, 5761 (1996) and references therein

[20] Y. Yamada, Phys. Rev. D 50, 3537 (1994).

[21] J. Hisano and M. Shifman, Phys. Rev. D 56, 5475 (1997).

[22] I. Jack and D. R. T. Jones, Phys. Lett. B 415, 383 (1997); I. Jack, D. R. T. Jones, and A. Pickering, Phys. Lett. B 426, 73 (1998); Phys. Lett. B 432, 114 (1998).

[23] D. I. Kazakov, and I. N. Kondrashuk, Nucl. Phys. B510, 289 (1998); D. I. Kazakov and V. N. Velizhanin, Phys. Lett. B 485, 393 (2000).

[24] T. Kobayashi, J. Kubo, and G. Zoupanos, Phys. Lett. B 427, 291 (1998).

[25] N. Arkani-Hamed, G. F. Giudice, M. A. Luty, and R. Rattazzi, Phys. Rev. D 58, 115005 (1998).

[26] See for a review, e.g., J. L. Feng, hep-ph/0405215.

[27] J. A. Casas, A. Lleyda, and C. Munoz, Nucl. Phys. B471, 3 (1996); Phys. Lett. B 380, 59 (1996); Phys. Lett. B 389, 305 (1996).

[28] B. A. Dobrescu and C. T. Hill, Phys. Rev. Lett. 81, 2634 (1998); R. S. Chivukula, B. A. Dobrescu, H. Georgi, and C. T. Hill, Phys. Rev. D 59, 075003 (1999); H. Collins, A. K. Grant, and H. Georgi, Phys. Rev. D 61, 055002 (2000).

[29] H. Nakano, in Strong Coupling Gauge Theories and Effective Field Theories edited by M. Harada, Y. Kikukawa, and K. Yamawaki (World Scientific, River Edge, 2003), p. 312. 